\title{
Editorial
}

\section{Applications of Molecular Methods in Human Medicine in India-Urgency to Bridge the Gap}

\author{
Vishwa Mohan Katoch \\ NASI-ICMR Chair on Public Health Research at RUHS, Jaipur, Former Secretary, Department \\ of Health Research, Govt of India and Director-General, Indian Council of Medical Research
}

It is well known that advances in technologies for molecular analysis have revolutionized all biological sciences including human medicine. While knowledge of biochemistry and cell biology has shown impact over a period of more than a century, capacity to map the changes at DNA/ RNA has led to development of specific and easy to use tools. Today, a huge number of techniques to identify genetic differences (natural diversity/gene mutations) are available and are being used to identify and characterize pathogens, to identify mutations responsible for diseases, and to map changes in entire genome and epigenome that are linked to health and disease. Clinicians and public health experts from all parts of world including India will like to use tools that can help in better strategies for diagnosis and management as well as prevention. We need to address whether India has done enough to harness the benefits of this vast knowledge that has become about genetic structure and function of large number of genomes of pathogens as well as humans.

At present several methods including PCR- SSCP, PCRRFLP, hybridization, and sequencing etc are available to identify mutations in a particular gene/gene region. Based on these technologies hundreds of methods for disease diagnosis of infectious/genetic disorders have been developed. Some of these technologies (molecular/ immunological) have been modified into point of care (POC) methods. Further, any new method based on need can be developed in a small time and that talent is in abundance in our country. Different sequencing methods targeting genes/gene regions allow for the identification of potential novel variants. They have their own advantages and limitations, one needs to analyse them according to his/ her own need.

Next-generation sequencing (NGS) technologies have ushered in a revolution in research as well as in practice of clinical medicine. These are excellent tools for identifying therapeutic targets and genomic markers for novel clinical applications-hereditary disorders, risk screening for hereditary cancers, and therapeutic decision making for somatic cancers and are not that expensive if their use is restricted. Whole Exome sequencing (WES) targets the complete coding region of the genome, estimated to encompass only approximately $1-2 \%$ of the genome, yet contains approximately $85 \%$ of disease-causing pathogenic variants. WES has been found to be quite useful in identifying new genes associated with rare diseases and cancers. Whole genome sequencing (WGS), the complete elucidation of the genomic determinants of a patient's heritable make-up, and thus is the most comprehensive tool for eliciting complete information. Studies have shown that NGS can be reliably applied on cytology specimens with high degree of sensitivity, specificity, and reproducibility. Successful applications include FNAC cytology specimens of cancers of colon, thyroid, lung, and pancreas. Oncologists have used them for classification for therapeutic purposes.

DNA chip and microarray technologies developed about 20-25 years ago opened the path for unraveling the structure and function of entire genome of all organisms. Over the years, the areas of structural and functional genomics as well as translational genomics have made rapid progress. Indian medical and biomedical researchers/practitioners need to analyse and understand the impact of new knowledge generated globally and at national level.

\section{Molecular biology in health and disease:}

Measuring the gene expression is important for research as well as management of many a human diseases. For this purpose real time PCR (RT-PCR) and microarrays have been found to be efficient approaches for quantitative and 
qualitative profiling of gene expression changes, however, their use is limited to the genes being assayed/ targeted. High-throughput gene expression profiling (GEP) using microarray technology was described more than 20 years ago and is a powerful tool to understand global gene expression changes. GEP has been found to be helpful in improving the classification and prognostication of non-Hodgkin Lymphomas (NHL), follicular lymphomas, and several others cancers. Its application has helped in better and rationale management of large number of patients suffering from these cancers. Refinement of the technology and analytical approaches has also enabled scientists to routinely evaluate practically the entire transcriptome at desired point of time which has been successfully used to improve our understanding of their pathophysiology and response to new therapeutics.

During the last 10-15 years, the development and use of the actionable gene panels has progressed in a big way. These are collections of well-studied actionable genes that are commonly involved in pathogenesis of several diseases. Most of such panels interrogate somatic mutations to aid in therapeutic decision-making, and assessing the risk of inherited diseases including cancers and have been found to be useful to diagnose suspected genetic diseases. More than 300 cancer panels have been developed, one third of which are available in commercial set ups. Some panels for hereditary diseases are also available.

Journey of molecular biology has moved from genetics to genomics to epigenetics and epigenomics. Epigenetics and epigenomics describe the alterations in gene expression without a change in nucleotide sequence in eukaryotes, these are mostly due to DNA methylation, miRNA and histone modification. These have been found to be useful to measure metabolic and environmental effects, impact of metabolism on the epigenome, how our actions may impact the health of our offspring, and how exposure to environmental toxicants can have longlasting effects on our epigenome with devastating consequences. There is explosion of discoveries related to the cellular regulation of phenotypes through epigenetic mechanisms associated with development of cancer and chemoresistance in cancer, diabetes and its complications, mental/neurological disorders, neonatal sepsis etc. Epigenetic and genetic abnormalities have been found to be of great value in understanding the mechanisms of cancer initiation and progression, as exemplified by frequent mutations in genes encoding proteins that control the epigenome. Many agents including phytochemicals targeting epigenetic regulation have become available to treat cancers, some are under development and are entering clinical trials. We need to use these approaches to understand the impact of natural factors like high fluoride content in water and simple dietary and other easy to use solutions to overcome health problems due to it.

\section{Translation potential:}

Innovations and their translation need to address the true needs of people specially those with low paying capacity. Affordable and efficient tools including diagnostics, vaccines, and therapeutics star getting larger population segments make better business sense as well. When we scan the data bases for genetics, genomics and epigenetics/ epigenomics, we find Indian presence. Good publications from India on various aspects are available. Indian scientists and laboratories have been pioneers in many a developments. However, Indian contributions comprise of hardly $1-1.5 \%$ of global data and the access for clinical applications is negligible. It is often the question of supply and demand. While many centres use the information about mutations to plan therapy in various cancers, it is difficult to understand why genomic based tools have been so rarely used for cancer management in India. Wider use will make them in-expensive and affordable for all. Such need will accelerate the commercial developments of such affordable technologies which will not only be useful for Indians but will help our biotechnology industry globally as well. Rajasthan, a respected state of Indian Union, is known for its historical rich past-valour, cultural diversity, and for many contributions in art and science. It is one of the rapidly changing states in India. Beginning in preindependence period and mostly during the last 70 years of independent India, state is known for establishment of many institutions of excellence. However, there are very few institutions in Rajasthan working on clinical applications of molecular biology in human medicine. There is an urgent need to discuss ideas to focus on innovation and translation as well as implementation of these technologies that will make an impact on the lives of people in Rajasthan, India and may be world at large. 